\title{
Target Centric Cyclic Pursuit using Bearing Angle Measurements Only
}

\author{
Sangeeta Daingade* Arpita Sinha** \\ * Systems and Control Engineering, IIT Bombay, Mumbai, India \\ (e-mail: sangeetad@sc.iitb.ac.in). \\ ** Systems and Control Engineering, IIT Bombay, Mumbai, India \\ (e-mail: asinha@sc.iitb.ac.in).
}

\begin{abstract}
This paper presents a cyclic pursuit based strategy for monitoring a stationary target with multiple autonomous vehicles. The strategy needs only bearing angle information of the target and that of only one of the neighbor vehicle. We have considered simple kinematic model for each vehicle. With the proposed control law, at equilibrium the vehicles get into a rigid polygonal formation which rotates around the target. The stability of equilibrium formations is analyzed using linearization technique. The effectiveness of the proposed strategy is demonstrated through simulation.
\end{abstract}

Keywords: Target monitoring, Multi - vehicle system, Bearing only measurements, Target centric cyclic pursuit.

\section{INTRODUCTION}

Multi vehicle systems offer many advantages over single vehicle mission in many military and civil applications. Such systems are proved to be more reliable, robust, scalable and also efficient. The multi vehicle systems are being used in applications like border patrolling, security systems, space and under water exploration and rescue operations. In order to accomplish a common goal these vehicles should work in cooperation. So we need to design suitable control laws depending on the type of application. The recent research topics in the area of cooperative control of multiple vehicles includes consensus, formation control, distributed task assignment and distributed estimation (refer Cao et al. (2013) and references therein). In this paper we address a problem of monitoring a target with multiple autonomous vehicles where the objective is to make the vehicles move around the target with uniform distribution.

Most of the target tracking strategies discussed in the literature (Paley et al. (2004), Klein and Morgansen (2006), Klein et al. (2007), Ceccarelli et al. (2008), Kingston and Beard (2008), Lan et al. (2010), Rattan and Ghosh (2009), Daingade and Sinha (2012)) are based on the position and/or velocity information about the neighbors which are defined in global reference frame. It would be an better idea if we can derive the control laws which are based on the quantities defined in the body frame of the vehicle. Moshtagh et al. (2009) have proposed a vision based control law for achieving circular formation which needs only bearing angle information of the neighbors. Each vehicle is assumed to have a vision sensor for measuring bearing angle which is a quantity defined in the local body frame. The agents finally converge to a circular formation but the point about which formation converges cannot be specified a priori. In order to enable target enclosing we should be able to achieve formations about a specific point (target).
Ground vehicle tracking using multiple UAVs considering vision input is discussed in Ma and Hovakimyan (2013) where the target tracking control and coordination control are designed separately. Here the tracking control is a function of both bearing angle and range measurements whereas coordination term in the control is a nonlinear function of bearing angle only.

In this paper we present a decentralized strategy for target enclosing with multiple vehicles, which needs only bearing angle information of one of the neighbor and the target. The novelty of the work lies in deriving a simple control law in terms of a quantity which can be easily measured and is defined in the local body frame. We study the case when the target is stationary. The main contributions of the paper are:

- Decentralized simple target tracking strategy for nonholonomic agents

- Control law is a linear function of bearing angles

- requires information of only one neighbor

- Stability analysis of the equilibrium formations.

It is assumed that all the vehicles can see the target. Also each vehicle can identify its neighbor vehicle and can measure bearing angle with respect to it. As the network topology is cyclic among the vehicles and the control law is designed considering neighbor vehicle as well as the target information, we call this strategy as Target Centric Cyclic Pursuit strategy. The paper is organized as follows. The system model with the proposed target centric law is presented in Sec 2 followed by the discussion about possible equilibrium formations in Sec 3. Stability analysis of these formations is studied in Sec 4 Simulation results are presented in Sec 5 and concluding remarks are discussed in 6 . 


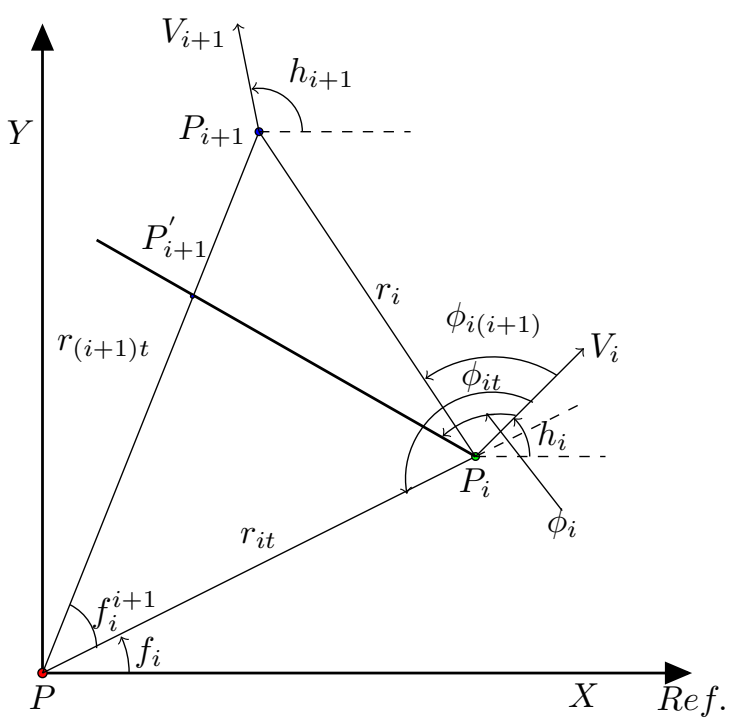

Fig. 1. Positions of the vehicles in a target centric frame

\section{PROBLEM FORMULATION}

Consider a group of $n$ vehicles employed to track a stationary target. We assume unicycle model for all the vehicles. Then the kinematics of each agent can be modeled as:

$$
\dot{x}_{i}=V_{i} \cos \left(h_{i}\right), \quad \dot{y}_{i}=V_{i} \sin \left(h_{i}\right), \quad \dot{h}_{i}=\omega_{i}
$$

where $P_{i}=\left[x_{i}, y_{i}\right]^{T}$ represents the position of agent $i$ and $h_{i}$ represents the heading angle of the agent $i . V_{i}$ and $\omega_{i}$ represents the linear speed and angular speed of agent $i$ respectively. Equation (1) can represent a point mass model of a UAV flying at a fixed altitude or a point mass model of a wheeled robot on a plane. We use a generic term "agent" to represent the aerial or ground vehicle. We assume that the agent $i$ is moving with constant linear speed, that is $V_{i}$ is constant and the motion of the agent $i$ is controlled using the angular speed, $\omega_{i}$. It is assumed that the agents are in cyclic pursuit. They are numbered from 1 to $n$. It is also assumed that all the vehicles can sense the target. So each vehicle has the bearing angle information of it's neighbor agent and also of the target. Consider Fig. 1. Point $P$ in the Fig. 1 represents the target position. Since we are considering a stationary target, we assume a target centric reference frame. Agent $i$ and its neighbor agent $i+1$ are located at $P_{i}$ and $P_{i+1}$ respectively. The variables in Fig. 1 are:

$r_{i t}$ - Distance between $i^{t h}$ agent and the target, $r_{i}$ - Distance between $i^{t h}$ agent and $i+1^{t h}$ agent, $f_{i}$ - angle made by the vector $r_{i t}$ w.r.t reference and $f_{i}^{i+1}$ - angular separation between agent $i$ and agent $i+1$ taken with respect to target.

$\phi_{i t}$ - Bearing angle of agent $i$ with respect to the target, $\phi_{i(i+1)}$ - Bearing angle of agent $i$ with respect to neighbor agent $i+1(\bmod n)$.

We modify the classical cyclic pursuit law (Marshall et al. (2004)) for target enclosing problem such that agent $i$, positioned at $P_{i}$, follows not only $i+1^{\text {th }}$ agent at $P_{i+1}$ but also the target at $P$. Let $\rho$ be a constant, which decides the weight agent $i$ gives to the target information, over the information of the agent $i+1$. The parameter $\rho$ can take values between 0 and 1 . This weighing scheme is mathematically equivalent to following a virtual leader along the line $P_{i} P_{i+1}^{\prime}$ with bearing angle $\phi_{i}$. The angle $\phi_{i}$ is calculated as

$$
\phi_{i}=(1-\rho) \phi_{i t}+\rho \phi_{i(i+1)} .
$$

We define the control input to the $i^{\text {th }}$ agent, as

$$
\omega_{i}=k_{i} \phi_{i}
$$

where, $k_{i}>0$ is the controller gain. We assume that $\phi_{i t} \in[0,2 \pi)$ and $\phi_{i(i+1)} \in[0,2 \pi)$ for all time, $t \geq 0$. So $\phi_{i} \in[0,2 \pi)$. This condition ensures that the agents always rotate in counter clockwise direction. The kinematics (1) can be re-written in the target centric reference frame as,

$$
\begin{aligned}
\dot{r}_{i t} & =V_{i} \cos \left(h_{i}-f_{i}\right) \\
\dot{f}_{i}^{i+1} & =\dot{f}_{i+1}-\dot{f}_{i} \\
& =\frac{V_{i+1} \sin \left(h_{i+1}-f_{i+1}\right)}{r_{(i+1) t}}-\frac{V_{i} \sin \left(h_{i}-f_{i}\right)}{r_{i t}} \\
\dot{h}_{i}-\dot{f}_{i} & =\omega_{i}-\dot{f}_{i}=k_{i} \phi_{i}-\frac{V_{i} \sin \left(h_{i}-f_{i}\right)}{r_{i t}}
\end{aligned}
$$

Let us define the states of the system as

$$
\begin{aligned}
& \mathbf{x}_{i(1)}=r_{i t} \\
& \mathbf{x}_{i(2)}=f_{i}^{i+1} \\
& \mathbf{x}_{i(3)}=h_{i}-f_{i}
\end{aligned}
$$

for $i=1,2, \cdots n$. Then we can write (4) - (6) as:

$$
\begin{aligned}
& \dot{\mathbf{x}}_{i(1)}=V_{i} \cos \left(\mathbf{x}_{i(3)}\right) \\
& \dot{\mathbf{x}}_{i(2)}=\frac{V_{i+1} \sin \left(\mathbf{x}_{i+1(3)}\right)}{\mathbf{x}_{i+1(1)}}-\frac{V_{i} \sin \left(\mathbf{x}_{i(3)}\right)}{\mathbf{x}_{i(1)}} \\
& \dot{\mathbf{x}}_{i(3)}=k_{i} \phi_{i}-\frac{V_{i} \sin \left(\mathbf{x}_{i(3)}\right)}{\mathbf{x}_{i(1)}} .
\end{aligned}
$$

Equation (8) gives the kinematics of $i^{\text {th }}$ agent. In the subsequent sections, all the analysis are done based on this model.

Note 1: In practical situation, the agents will have a bound on angular speed $\omega_{\max }$, that is, $\omega_{i} \leq \omega_{\max } \forall i$. We can take into account this constraint by imposing an upper bound on the value of $k_{i}$ as, $k_{i} \leq k_{\max }$ where $k_{\max }=\frac{\omega_{\max }}{2 \pi}$.

Next section discusses about the possible formations at equilibrium.

\section{FORMATION AT EQUILIBRIUM}

In this section, we study the asymptotic behavior of the agents under the control law (3).

Theorem 1. Consider $n$ agents with kinematics (8) and control law (3). At equilibrium, the agents move on a concentric circles, with $(i)$ the target at the center of concentric circles and $(i i)$ equal angular velocities.

Proof. At equilibrium $\dot{\mathbf{x}}_{i(j)}=0$ for $i=1, \ldots, n$ and $j=1,2,3$, which implies, from (7), that

$$
\begin{aligned}
\dot{r}_{i t} & =0 \\
\dot{f}_{i}^{i+1} & =0 \\
\dot{h}_{i}-\dot{f}_{i} & =0
\end{aligned}
$$

Then, from (4) - (6), at equilibrium

$$
\begin{aligned}
& \mathbf{x}_{i(1)}=r_{i t}=\text { constant } \\
& \mathbf{x}_{i(2)}=f_{i}^{i+1}=\text { constant } \\
& \mathbf{x}_{i(3)}=h_{i}-f_{i}=\text { constant }
\end{aligned}
$$


From (12), we observe that the distance between the target and agent $i$ (for all $i$ ) remains constant at equilibrium. Using (4) and (9) we can write,

$$
h_{i}-f_{i}=(2 m+1) \frac{\pi}{2}
$$

where $m=0, \pm 1, \pm 2, \cdots$. From (6), (11) and (15),

$$
k_{i} \phi_{i}=\frac{V_{i} \sin \left(h_{i}-f_{i}\right)}{r_{i t}}= \pm \frac{V_{i}}{r_{i t}}
$$

Since $k_{i}>0, V_{i}>0$ and $r_{i t} \geq 0$, from (16) we get

$$
k_{i} \phi_{i}=\frac{V_{i}}{r_{i t}}
$$

and therefore, in (15), $m=0, \pm 2, \pm 4, \cdots$. Assuming $h_{i} \in$ $[0,2 \pi)$ and $f_{i} \in[0,2 \pi)$, we get $\left(h_{i}-f_{i}\right) \in(-2 \pi, 2 \pi)$. Therefore $m=0$ or $m=-2$. From geometry, $m=0$ and $m=-2$ implies the same angle. Therefore

$$
h_{i}-f_{i}=\frac{\pi}{2}
$$

From (3) and (17),

$$
\omega_{i}=\frac{V_{i}}{r_{i t}}
$$

Since $V_{i}$ and $r_{i t}$ are constant, $\omega_{i}$ is constant for all $i$. Therefore all the agents move along a circular path with the target at the center and radius $r_{i t}$. This proves the first part of the theorem.

From equation (5), (10) and (18), we can write,

$$
\frac{V_{i}}{r_{i t}}=\frac{V_{i+1}}{r_{(i+1) t}}
$$

Using (19) and (20), we conclude that

$$
\omega_{i}=\omega_{i+1}
$$

for all $i$. Therefore, all the agents move around the target in concentric circles with equal angular speed.

Corollary 2. Consider $n$ agents with kinematics (8). At equilibrium, the agents form a rigid polygon that rotates about the target.

Proof. The proof follows directly from the Theorem 1

In this paper we present analysis for homogeneous agents only. The agents are assumed to be homogeneous in the sense that all of them move with equal linear speed $V$ and equal controller gain $k$. As $V_{i}=V_{i+1}$, from (20), $r_{i t}=r_{(i+1) t}=R$ for all $i$. Therefore at equilibrium all the agents move along a circle of radius $R$ with the target at the center. Consider Fig. 2 which shows the position of two of the agents at equilibrium. Let $P, P_{i}$ and $P_{i+1}$ be the positions of target, agent $i$ and agent $i+1$ respectively. From Fig. $1, \phi_{i t}=\pi-\left(h_{i}-f_{i}\right)$. Substituting the value of $h_{i}-f_{i}$ from (18),

$$
\phi_{i t}=\frac{\pi}{2}
$$

for all $i$. Consider $\triangle P_{i} P P_{i+1}$. As $P_{i} P=P_{i+1} P=R$, $\angle P P_{i} P_{i+1}=\angle P P_{i+1} P_{i}=b_{i}$. Therefore $f_{i}^{i+1}=\pi-2 b_{i}$. Referring Fig. 2 and using (22), we can write $b_{i}=\frac{\pi}{2}-$ $\phi_{i(i+1)}$. So

$$
\phi_{i(i+1)}=\frac{f_{i}^{i+1}}{2} .
$$

From (3), (19) and (21) we can write,

$$
\phi_{i}=\phi_{i+1}=\frac{V}{k R} \text {. }
$$

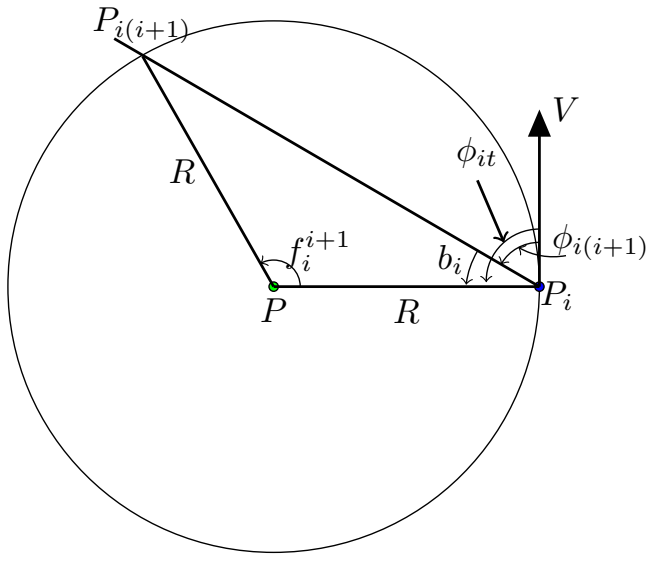

Fig. 2. Formation of agent $i$ and agent $i+1$ at equilibrium

So using (2), (22), (23) and (24) we can conclude that $f_{i}^{i+1}=f_{i+1}^{i+2}$ for all $i$ which implies that the agents are uniformly distributed around target. As the agents are distributed along a circle, $\sum_{(\bmod \mathrm{n})}^{n}\left(f_{i}^{i+1}\right)=2 \pi d$, where $d=0, \pm 1, \pm 2, \cdots$. Therefore we can write,

$$
f_{i}^{i+1}=\frac{2 \pi d}{n}
$$

for all $i$. Thus the value of $\phi_{i}$ at equilibrium can be calculated by using (2), (22), (23) and (25) as,

$$
\phi_{e q}=(1-\rho) \frac{\pi}{2}+\rho \frac{\pi d}{n} .
$$

Then the equilibrium state of $n$ agent system can be described as

$$
\begin{aligned}
& \mathbf{x}_{i(1)}=R=\frac{V}{k \phi_{e q}} \\
& \mathbf{x}_{i(2)}=2 \pi \frac{d}{n} \\
& \mathbf{x}_{i(3)}=\frac{\pi}{2} .
\end{aligned}
$$

The inter agent distance $R_{a a}$, can be obtained (refer to Fig. 2) as

$$
R_{a a}=2 R \sin \left(\frac{\pi d}{n}\right)
$$

Thus, at equilibrium, the agents arrange themselves in a regular formation around the target. This regular formation of $n$ agents can be described by a regular polygon $\{n / d\}$, where $d \in\{1,2, \ldots, n-1\}$. This $d$ is reflected in equilibrium state $\mathbf{x}_{i(2)}$ in $(27 \mathrm{~b})$. The regular polygon $\{n / d\}$ is defined as follows. If $Z_{i}$ is the point on the circle and $\delta_{r}$ is clockwise rotation by an angle $2 \pi \frac{d}{n}$ along the circle from point $Z_{i}$, then the regular polygon is defined by the set of vertices given by $Z_{i+1}=\delta_{r} Z_{i}$, and edges between $Z_{i+1}$ and $Z_{i}$. When $d=1$, then polygon $\{n / d\}$ is called an ordinary regular polygon, and its edges do not intersect. If $d>1$ and if $n$ and $d$ are coprime, then the edges intersect, and the polygon is a star. If $n$ and $d$ have a common factor $c_{m}>1$, then the polygon consists of $c_{m}$ traversals of the same polygon with $n / c_{m}$ vertices and $n / c_{m}$ edges. In the next section we study the stability of these formations.

\section{STABILITY ANALYSIS}

In this section, we study the stability of the equilibrium formations. With $n$ number of agents in the system, the 
equilibrium state is described by (27a)-(27c). Since $d$ can take $(n-1)$ values, there are total $(n-1)$ formations possible for a given value of $n$. Linearizing the system (8) about the equilibrium point (27a)-(27c), we get a linear model of agent $i$ as

$$
\dot{\hat{\mathbf{x}}}_{i}=A \hat{\mathbf{x}}_{i}+B \hat{\mathbf{x}}_{i+1}
$$

where $A$ and $B$ are,

$$
\begin{aligned}
& A=\left[\begin{array}{ccc}
0 & 0 & -V \\
\frac{V}{R^{2}} & 0 & 0 \\
\frac{k \rho \cot \left(\frac{\pi d}{n}\right)}{2 R}+\frac{V}{R^{2}} & \frac{k \rho}{2} & -k
\end{array}\right] \\
& B=\left[\begin{array}{ccc}
0 & 0 & 0 \\
\frac{-V}{R^{2}} & 0 & 0 \\
\frac{-k \rho \cot \left(\frac{\pi d}{n}\right)}{2 R} & 0 & 0
\end{array}\right]
\end{aligned}
$$

So, the system of $n$ agents, can be written as

$$
\dot{\hat{X}}=\hat{A} \hat{X}
$$

where $\hat{X}=\left[\hat{\mathbf{x}}_{1}, \hat{\mathbf{x}}_{2}, \cdots, \hat{\mathbf{x}}_{n}\right]^{T}, \hat{\mathbf{x}}_{i}=\left[\mathbf{x}_{i(1)}, \mathbf{x}_{i(2)}, \mathbf{x}_{i(3)}\right]^{T}$ and $\hat{A}$ is a circulant matrix given by $\hat{A}=\operatorname{circ}\left(A B 0_{3 \times 3} \cdots 0_{3 \times 3}\right)$ The stability of the formation depends on the eigen values of $\hat{A}$.

Theorem 3. Consider $n$ agents with kinematics (8). For a given value of $\rho$, the equilibrium points given by (27) are locally asymptotically stable if

$$
q_{l}<\frac{d}{n}<q_{u}
$$

where,

$$
\begin{gathered}
q_{l}=\left\{\begin{array}{lc}
0.13 \rho+0.01 & \text { for } \rho \leq 0.8 \\
0.114 \sin ^{8}\left(\frac{(\rho+0.2) \pi}{2}\right) & \text { for } \rho>0.8
\end{array}\right. \\
q_{u}=\left\{\begin{array}{lc}
\sqrt{\frac{2}{\pi} \cos ^{-1}(\rho)} & \text { for } \rho \leq 0.8 \\
\max \left(\sqrt{\frac{2}{\pi} \cos ^{-1}(\rho)}-0.5 \sin \left(\frac{(\rho-0.8) \pi}{2}\right), 0\right) & \text { for } \rho>0.8
\end{array}\right.
\end{gathered}
$$

Proof. The stability of a formation depends on the eigen values of the circulant matrix $\hat{A}$. We can find the eigen values of block circulant matrix $\hat{A}$, (as given in Davis (1994)) by block diagonalizing the system matrix using Fourier matrix $F_{n}$. The Fourier matrix $F_{n}$ is given by,

$$
F_{n}=\frac{1}{\sqrt{n}}\left[\begin{array}{ccccc}
1 & 1 & 1 & 1 & 1 \\
1 & \varsigma & \varsigma^{2} & \ldots & \varsigma^{n-1} \\
\vdots & \vdots & \vdots & \vdots & \vdots \\
1 & \varsigma^{n-1} & \varsigma^{2(n-1)} & \ldots & \varsigma^{(n-1)(n-1)}
\end{array}\right]
$$

where $\varsigma=e^{j \frac{2 \pi}{n}}$ and $j=\sqrt{-1}$. Then the block circulant matrix $\hat{A}$ can be diagonalized as,

$$
\hat{A}=\left(F_{n} \otimes I_{3}\right) D\left(F_{n} \otimes I_{3}\right)^{\star}
$$

where $(\star)$ indicates conjugate transpose. The diagonal matrix $D$ is given by,

$$
D=\operatorname{diag}\left(D_{1}, D_{2}, \cdots, D_{n}\right) .
$$

where $D_{i}=A+\varsigma^{i-1} B, i \in\{1,2, \ldots . n\}$ and

$$
D_{i}=\left[\begin{array}{ccc}
0 & 0 & -V \\
\frac{V}{R^{2}}\left(1-\varsigma^{i-1}\right) & 0 & 0 \\
\frac{k \rho \cot \left(\frac{\pi d}{n}\right)\left(1-\varsigma^{i-1}\right)}{2 R}+\frac{V}{R^{2}} & \frac{k \rho}{2} & -k
\end{array}\right] .
$$

The eigen values of $\hat{A}$ are same as eigenvalues of $D_{i}$, $i=1, \cdots n$. So we can comment about the stability of $n$ vehicle system, by observing the eigen values of each block $D_{i}$. After substituting the value of $R$ from (27a), each $D_{i}$ can be factorized as $D_{i}=k T \tilde{D}_{i} T^{-1}$, where $T=\operatorname{diag}\left[\frac{1}{k \phi_{e q}}, \frac{1}{V}, \frac{1}{V}\right]$ and

$$
\tilde{D}_{i}=\left[\begin{array}{ccc}
0 & 0 & -\phi_{e q} \\
\phi_{e q}\left(1-\varsigma^{i-1}\right) & 0 & 0 \\
\frac{\rho \cot \left(\frac{\pi d}{n}\right)\left(1-\varsigma^{i-1}\right)}{2}+\phi_{e q} & \frac{\rho}{2} & -1
\end{array}\right]
$$

The spectrum $\sigma(\cdot)$ of $D_{i}$ and $\tilde{D}_{i}$ are related as $\sigma\left(D_{i}\right)=$ $k \sigma\left(\tilde{D}_{i}\right)$. Since $k>0$, stability of $D_{i}$ can be determined from the stability of $\tilde{D}_{i}$. $\tilde{D}_{i}$ does not have a term containing gain $k$. So we can conclude that the stability of $\{n / d\}$ formation is independent of $k$, as long as $k>0$.

$\tilde{D}_{i}$ is a complex matrix, as $\varsigma^{i-1}=e^{2 \pi j \frac{i-1}{n}}$ is a complex quantity. We can write $\varsigma^{i-1}=\beta_{i}+j \xi_{i} \in \mathbb{C}$, where $\beta_{i}=$ $\cos \left(2 \pi \frac{i-1}{n}\right)$ and $\xi_{i}=\sin \left(2 \pi \frac{i-1}{n}\right)$. Then the characteristic polynomial of $\tilde{D}_{i}$ can be written as $P_{\tilde{D}_{i}}(\lambda)=\lambda^{3}+c_{1} \lambda^{2}+$ $c_{2} \lambda+c_{3}$, where $c_{1}=1, c_{2}=a_{2}+j b_{2}, c_{3}=a_{3}+j b_{3}$ and,

$$
\begin{aligned}
a_{2} & =\frac{\phi_{e q} \rho}{2} \cot \left(\pi \frac{d}{n}\right)\left(1-\beta_{i}\right)+\phi_{e q}^{2} \\
b_{2} & =\frac{\phi_{e q} \rho}{2} \cot \left(\pi \frac{d}{n}\right)\left(-\xi_{i}\right) \\
a_{3} & =\frac{\phi_{e q}^{2} \rho}{2}\left(1-\beta_{i}\right) \\
b_{3} & =\frac{\phi_{e q}^{2} \rho}{2}\left(-\xi_{i}\right)
\end{aligned}
$$

Define the Hermitian matrix $H$ for the above characteristic polynomial $P_{\tilde{D}_{i}}(\lambda)$ as,

$$
H=\left[\begin{array}{ccc}
c_{1}+\bar{c}_{1} & c_{2}-\bar{c}_{2} & c_{3}+\bar{c}_{3} \\
-c_{2}+\bar{c}_{2} & c_{2}+\bar{c}_{2}-c_{3}-\bar{c}_{3} & c_{3}-\bar{c}_{3} \\
c_{3}+\bar{c}_{3} & -c_{3}+\bar{c}_{3} & c_{2} \bar{c}_{3}+\bar{c}_{2} c_{3}
\end{array}\right] .
$$

Then the polynomial $P_{\tilde{D}_{i}}(\lambda)$ is asymptotically stable if and only if the principal minors $h_{1}, h_{2}$ and $h_{3}$ of $H$ are positive Barnett (1993). In this case

$$
\begin{aligned}
h_{1}= & 2 \\
h_{2}= & 4\left(a_{2}-a_{3}-b_{2}{ }^{2}\right) \\
h_{3}= & 8\left(a_{2}{ }^{2} a_{3}+a_{2} b_{2} b_{3}-2 a_{2} a_{3}{ }^{2}-3 a_{3} b_{2} b_{3}\right. \\
& \left.-b_{3}{ }^{2}-a_{3} b_{2}{ }^{2} a_{2}-b_{2}{ }^{3} b_{3}+a_{3}{ }^{3}\right)
\end{aligned}
$$

Here, $h_{2}$ and $h_{3}$ depends on $\frac{d}{n}$ and $\beta_{i}$ which takes discrete values. To find the range of values of $\frac{d}{n}$ and $\beta_{i}$ for which $h_{2}>0$ and $h_{3}>0$, we replace $\frac{d}{n}$ by a continuous variable $q \in(0,1)$. Also we replace $\beta_{i}$ by a continuous variable $\tilde{\beta} \in[-1,1]$. For a given $\tilde{\beta}$, we can plot $q$ versus $\rho$ when $h_{2}=0$ and also when $h_{3}=0$. Fig. 3 shows these plots for different values of $\tilde{\beta}$. Let us define $S_{2}$ and $S_{3}$ as:

$$
S_{2}=\left\{(\rho, q, \tilde{\beta}): h_{2}>0, \rho \in(0,1), q \in(0,1), \tilde{\beta} \in[-1,1]\right\}
$$$$
S_{3}=\left\{(\rho, q, \tilde{\beta}): h_{3}>0, \rho \in(0,1), q \in(0,1), \tilde{\beta} \in[-1,1]\right\}
$$

Then $S=S_{2} \cap S_{3}$, defines the stability region where both $h_{2}$ and $h_{3}$ are positive. In Fig. 3, Region $I$ corresponds 


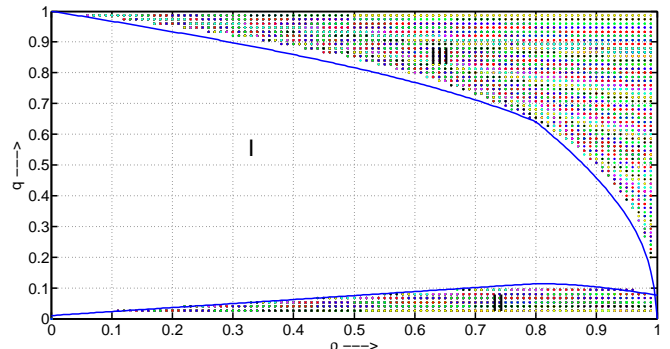

Fig. 3. Stability region for continuous values of $q, \rho$ and $\tilde{\beta}$ (Dotted lines represent locus of points where $h_{2}=0$ or $\left.h_{3}=0\right)$

Table 1. Comparison between Analytical and and Simulation results

\begin{tabular}{|c|c|c|c|c|c|}
\hline & & \multicolumn{2}{|c|}{$R$} & \multicolumn{2}{c|}{$R_{a a}$} \\
\hline InitialCond. & $\mathrm{d}$ & Analytical & Simulation & Analytical & Simulation \\
\hline 1 & 2 & 121.5365 & 123.5365 & 190.0241 & 190.0241 \\
\hline 2 & 3 & 102.8386 & 102.8383 & 200.5204 & 200.5203 \\
\hline 3 & 4 & 89.1268 & 89.1268 & 173.7843 & 173.7843 \\
\hline
\end{tabular}

to $S$. We can numerically approximate the Region $I$ with conservative bounds and can define the bounds on $q$ as be defined by (33). Thus we can conclude that for a given $\rho$ and $n$, if $\frac{d}{n}$ satisfies (33), then the eigen values of $\tilde{D}$ and hence that of $\hat{A}$ will have negative real part. So the corresponding formation will be asymptotically stable.

\section{SIMULATION RESULTS}

We consider seven agents all moving with a linear speed of $15 \mathrm{~m} / \mathrm{sec}$ and having controller gain $k=0.1$. The target is stationary and is located at the origin $(0,0)$.

Case 1: In this case agents start from random initial positions with $\rho=0.5$. Fig. 4(a) shows the trajectories of the agents at equilibrium. The agents finally converge to $\{7 / 4\}$ formation about the target point. The plots in Fig. 4 (b) and Fig. 4(c), confirms the inter agent distance, $\left(R_{a a}\right)$ and the distance between the agents and the target $(R)$, evaluated analytically using (28) and (27a). Table 1 shows the calculated and simulated values of these distances for three different initial conditions resulting in three different formations. From the Table 1, we observe that the analytical results match with those obtained using simulation. The final formation is a function of initial positions of agents, linear speed of the agents, controller gain and the parameter $\rho$.

In next two cases, we select $\rho$ and initial $d$ such that the agents are initially at equilibrium and the equilibrium points lies in one of the sector $I$ and $I I I$ of Fig. 3.

Case 2: Consider seven agents initially in $\{7 / 2\}$ formation with $\rho=0.5$. Here $R=121.5365$ initially and this formation corresponds to a point in Section $I$ in Fig. 3. As $q_{l}=0.0750, q_{u}=0.8165$ and $\frac{d}{n}=0.2857$, Theorem 3 is satisfied. So this is a case of stable formation. Fig. 5(a) shows that, at equilibrium it stays in $\{7 / 2\}$ formation and confirms the analytical results.

Case 3: In this case, the agents are initially in $\{7 / 6\}$ formation and are distributed along a circle of radius $R=70.3632$ with target at the center. The value of $\rho=0.5$. From (33), the limits for $\frac{d}{n}$ are $q_{l}=0.0750$ and $q_{u}=0.8165$. As $\frac{d}{n}=6 / 7=0.8571$, it corresponds to a

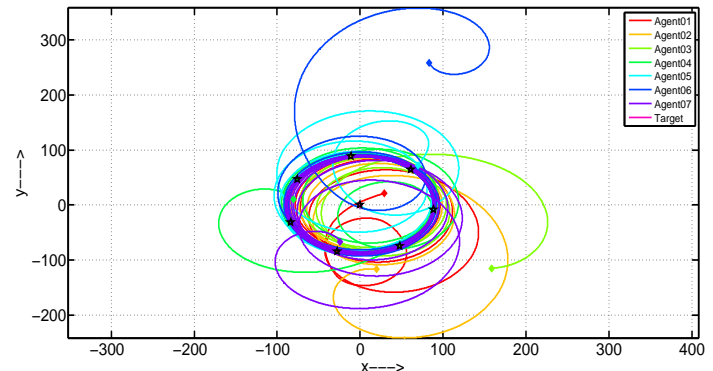

(a) Trajectories of agents $(\diamond-$ initial position, $\star-$ final position of the agents)

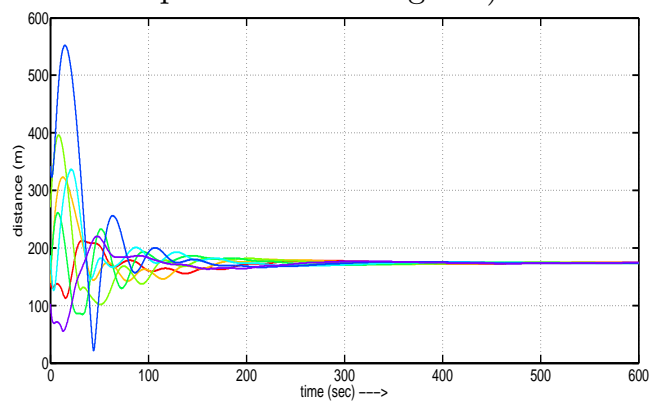

(b)Inter agent distance with respect to time

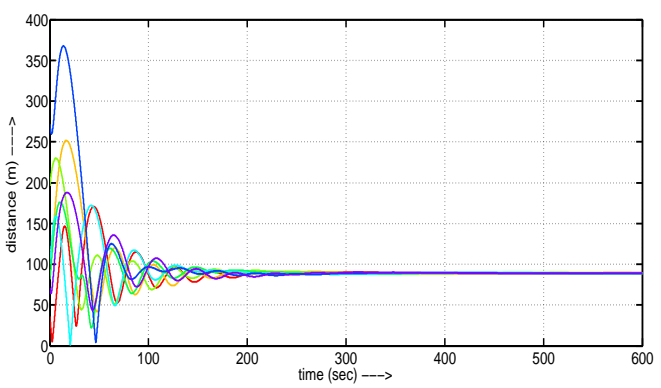

(c) Agent to target distance with respect to time

Fig. 4. Seven agents in formation

point in Section III of Fig. 3. As Theorem 3 is violated, this is a case of unstable formation. Fig. 5(b) shows that, even though the agents are initially in $\{7 / 6\}$ formation, the system finally settles with $\{7 / 3\}$ formation. As shown in Table 1, analytical and simulated values of $R$ and $R_{a a}$ are same at equilibrium. The $\{7 / 3\}$ formation for $\rho=0.5$ corresponds to Section $I$ of Fig. 3 .

Lastly, we show that this strategy also works well for nonstationary target.

Case 4: Consider a target, moving linearly with a speed of $1.5 \mathrm{~m} / \mathrm{sec}$ and $\rho=0.4$. The agents start from random initial positions. Fig. 6 show that they get into a $7 / 4$ formation about the moving target and continue to enclose it maintaining the formation. This illustrates the potential of the proposed strategy for cooperatively tracking a slow moving target.

Next we considered five heterogeneous agents moving with different linear speed ( $V=10,13,18,20,16 \mathrm{~m} / \mathrm{sec})$. They all move with $\rho=0.6$ and $k=0.1$. Fig. 7 shows the trajectories of all the agents. It is observed that the agents finally move along concentric circles with the target at the center. 


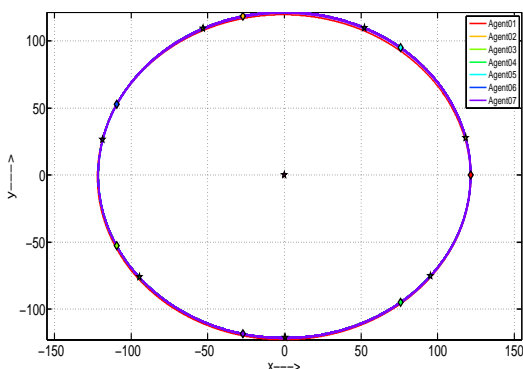

(a) Case 2: Initial formation $\{7 / 2\}$, final formation $\{7 / 2\}$

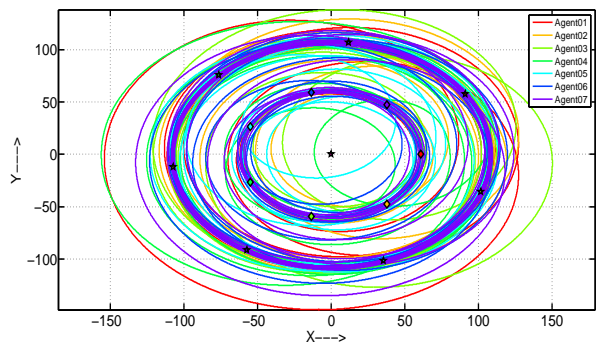

(b) Case 3: Initial formation $\{7 / 6\}$, final formation $\{7 / 3\}$

Fig. 5. Stable and unstable formation of seven homogeneous agents $(\diamond-$ initial position, $\star$ - final position of the agents)

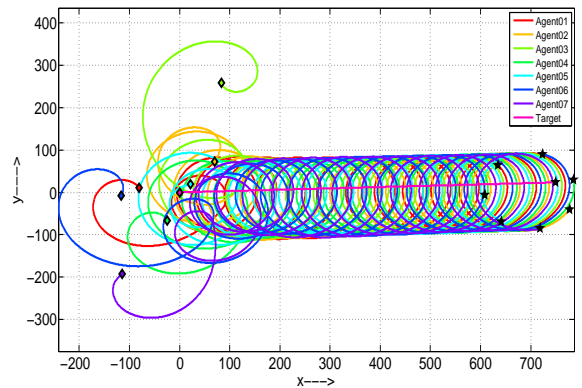

Fig. 6. Agents tracking a moving target $(\diamond-$ initial position, $\star$ - final position of the agents)

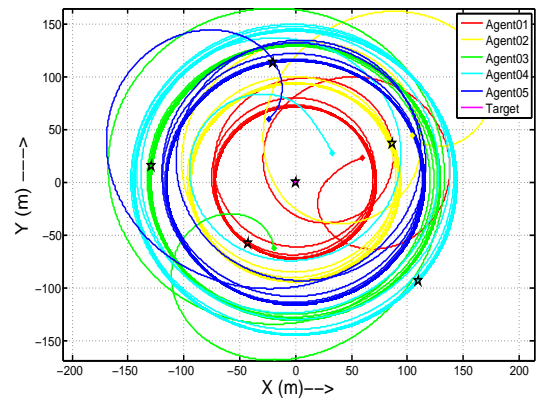

Fig. 7. Trajectories of 5 heterogeneous agents $(\diamond-$ initial position, $\star-$ final position of the agents)

\section{CONCLUSIONS}

In this paper we proposed bearing only target centric control law for target monitoring using a group of heterogeneous agents. The agents are modeled as planar unicycles moving with constant speed. The analysis has been carried out in details for a stationary target. At equilibrium, the agents move along concentric circles with the target at the center. When the agents are identical they move along a circle with a uniform distribution around the target. There are total $(n-1)$ polygonal formations possible at equilibrium. The type of the formation depend on initial conditions and control parameter. Stability analysis of these equilibrium formations based on linearization technique shows that the parameter $\rho$ plays an important role in the formation achieved at the equilibrium. Simulation results match with the results obtained analytically. With the simulation it has been shown that the proposed strategy works well in case of a moving target.

\section{REFERENCES}

Barnett, S. (1993). Polynomials and linear control systems. Monographs and textbooks in Pure and Applied Mathematics Series.

Cao, Y., Yu, W., Ren, W., and Chen, G. (2013). An overview of recent progress in the study of distributed multi-agent coordination. IEEE Transactions on Industrial Informatics, 09(01), 427-438.

Ceccarelli, N., Marco, M.D., Garulli, A., and Giannitrapani, A. (2008). Collective circular motion of multivehicle systems. Automatica, 44(12), 3025-3035.

Daingade, S. and Sinha, A. (2012). Nonlinear cyclic pursuit based cooperative target monitoring. International Symposium on Distributed Autonomous Robotic Systems (DARS-2012).

Davis, P.J. (1994). Circulant Matrices. NewYork, Chelsea, second edition.

Kingston, D. and Beard, R. (2008). Uav splay state configuration for moving targets in wind. Advances in coperative control and optimization, Lecture notes in Computer Science, 109-128.

Klein, D.J., Matlack, C., and Morgansen, K.A. (2007). Cooperative target tracking using oscillator models in three dimensions. Proceedings of the American Control Conference, 2569-2575.

Klein, D.J. and Morgansen, K.A. (2006). controlled collective motion for mulitvehicle trajectory tracking. Proceedings of the American Control Conference, 52695275.

Lan, Y., Yan, G., and Lin, Z. (2010). Distributed control of cooperative target enclosing based on reachability and invariance analysis. Systems \& Control Letters, 59(07), 381-389.

Ma, L. and Hovakimyan, N. (2013). Cooperative target tracking in balanced circular formation: Multiple uavs tracking a ground vehicle. Proceedings of the American Control Conference, 5386-5391.

Marshall, J.A., Broucke, M.E., and Francis, B.A. (2004). Formations of vehicles in cyclic pursuit. IEEE Transaction on Automatic Control, 49(11), 1963-1974.

Moshtagh, N., Michael, N., Jadbabaie, A., and Daniilidis, K. (2009). Vision-based, distributed control laws for motion coordination of nonholonomic robots. IEEE Transactions on Robotics, 25(04), 851-860.

Paley, D., Leonard, N., and Sepulchre, R. (2004). Collective motion: bistability and trajectory tracking. Proceedings of the 43rd IEEE Conference on Decision and Control, 1932-1937.

Rattan, G. and Ghosh, D. (2009). Nonlinear cyclic pursuit strategies for mav swarms. Technical Report, DRDOIISc Programme on Advanced Research in Mathematical Engineering, 1-32. 